\title{
summary
}

\section{No conclusive evidence for caries-inhibitory effect of glass-ionomer restorations in vivo}

Randall RC, Wilson NHF. Glass-ionomer restoratives: a systematic review of a secondary caries treatment effect. J Dent Res, 1999; 78:628-637

Objective To determine from a literature analysis the ability of glass-ionomer restorations to prevent recurrent caries at the restorative margins.

Data sources Literature searches covering articles (1970-1996) from Medline and Exerpta Medica databases, from databases in dental school libraries at Nijmegen and Gothenburg, and from hand searches of English and German dental journals.

Selection criteria Fifty-two studies were identified but none complied with the 14 primary inclusion criteria. Using a secondary set of two criteria ('prospective studies with controls') 28 articles were selected for the analysis.

Results The 28 selected articles were grouped into four categories according to findings of secondary caries in either the glass-ionomer or the control groups (see Table 1).

\begin{tabular}{cccc}
\multicolumn{4}{l}{ Table 1 Secondary caries findings } \\
\hline Category & Studies $(\mathrm{n})$ & Glass ionomer & Control \\
\hline 1 & 5 & No secondary caries & Secondary caries \\
2 & 10 & No secondary caries & No secondary caries \\
3 & 4 & Secondary caries & No secondary caries \\
4 & 9 & Secondary caries & Secondary caries \\
\hline
\end{tabular}

Conclusions The review of the literature did not produce conclusive evidence for the generally accepted effect of secondary caries inhibition by glass-ionomer restorations.

Address for reprints: RC Randall, Restorative Dentistry, Manchester University, Turner Dental School, Higher Cambridge Street, Manchester M15 6FH, UK.

\section{Commentary}

There are ample data from in vitro studies on the caries-inhibitory effect of glass-ionomer restorations, but a similar in vivo effect is less evident in the literature and research reports. In this comprehensive analysis of existing literature the authors attempted to find evidence that would either confirm or dismiss the accepted premise that glass-ionomer restorations can inhibit secondary caries in patients. A reasonable number of relevant articles (52) were found in the English and German literature that could provide information pertinent to the review. By applying a set of 14 selection criteria by which the authors expected to identify the most appropriate articles, however, they found that none of the articles complied with all criteria. In retrospect, the selected criteria may have included items that were unnecessarily restrictive, eg. the criterion requiring $100 \%$ patient recall, a parameter that can be difficult to fulfil in longitudinal clinical studies. Still, it was somewhat alarming to notice that the majority of clinical studies identified for this project did not comply with established standards for design and reporting of clinical research. This necessitated a lowering of the standard of selection criteria to allow completion of the project. The new selection criteria included only two requirements: first, that the research was a prospective study and second, there was a control group.

In grouping the articles in categories according to findings of secondary caries the authors found a simple method to compare the outcomes. The grouping clearly demonstrates the conflicting findings by various researchers from studies with 'no recurrent caries' in any groups to studies with 'caries' findings in both groups. The total number of studies with caries findings in the glass-ionomer group $(n=13)$ is close to the number of reports with caries in the control group $(\mathrm{n}=14)$.

The use of only four categories may have been somewhat simplistic. In category 4 (caries in both groups) there were several studies that found a higher incidence of caries in the control group ( $14 \%$ versus $2 \%, 10 \%$ versus $3 \%$ ). These could be assigned to a different category. Indeed, the authors performed additional analyses of the data by comparing subgroups of studies with unique characteristics, eg. studies with high-caries-risk patients or studies with large sample sizes, but there were no significant differences from the main effect.

An interesting observation in this metaanalysis is the difference in caries-rates findings between studies. A 10-year clinical study reported no secondary caries in either group, whereas a 3-year and a 5-year study had caries findings of $10 \%$ and $11 \%$, respectively, in the composite control group. These differences can be attributed to different study populations, effect of operator and techniques, and different study design and evaluation criteria. One limitation of the analysis may be an inherent difference between the results from controlled clinical studies and the profession at large. The relatively low incidence of recurrent caries reported in these studies may be indicative of a study effect: when outcomes are measured carefully and speed of performance is not an issue, operators tend to be far more thorough in placing the restorations.

The study concludes that there was no conclusive evidence in the literature for or against the in vivo caries-inhibitory effect of glassionomer restorations. The authors also remarked that there was a need to reassess the current clinical-evaluation methodology of glass-ionomers for more conclusive future reviews.

Dan Nathanson Department of Biomaterials, Goldman School of Dental Medicine, Boston University, Boston, Massachusetts, USA 